Uma Análise Descritiva sobre o Processo de Transferência de Conhecimento dos Trabalhadores Maduros para os mais Jovens nas Organizações

Dissertação apresentada ao Programa de Pósgraduação em Administração de Empresas da PUC-Rio como requisito parcial para obtenção do titulo de Mestre em Administração de Empresas.

Orientadora: Prof. a Sandra Regina da Rocha-Pinto 
Danielle Didier Lyra

\section{Uma Análise Descritiva sobre o Processo de Transferência de Conhecimento dos Trabalhadores Maduros para os mais Jovens nas Organizações}

Dissertação apresentada como requisito parcial para obtenção do grau de Mestre pelo programa de Pósgraduação em Administração de Empresas da PUC-Rio. Aprovada pela Comissão Examinadora abaixo assinada.

Prof.- Sandra Regina da Rocha

Orientadora

Departamento de Administração, PUC-Rio

Prof. Departamento de Administração, PUC-Rio

Prof.- Maria Teresa Correia Coutinho Departamento de Administração, FACC-UFRJ

Profa. Monica Herz

Vice-Decana de Pós-Graduação do CCS

Rio de Janeiro, 27 de outubro de 2009 
Todos os direitos reservados. É proibido a reprodução total ou parcial do trabalho sem autorização da universidade, da autora e da orientadora.

\section{Danielle Didier Lyra}

Graduou-se em Administração com ênfase em Marketing na UFRJ (Universidade Federal do Rio de Janeiro) em 2007

Ficha Catalográfica

Lyra, Danielle Didier

Uma análise descritiva sobre o processo de transferência de conhecimento dos trabalhadores maduros para os mais jovens nas organizações / Danielle Didier Lyra ; orientadora: Sandra Regina da Rocha-Pinto. $-2009$.

144 f. ; $30 \mathrm{~cm}$

Dissertação (Mestrado em Administração)Pontifícia Universidade Católica do Rio de Janeiro, Rio de Janeiro, 2009.

Inclui bibliografia

1. Administração - Teses. 2. Gestão do conhecimento. 3. Memória organizacional. 4. Trabalhador maduro. I. Rocha-Pinto, Sandra Regina da. II. Pontifícia Universidade Católica do Rio de Janeiro. Departamento de Administração. III. Título. 


\section{Agradecimentos}

A Jesus autor da vida, fonte de alegria, paz, força e inspiração.

À minha família: aos meus pais pelo incansável apoio, conselhos, incentivos e investimento na minha educação. Ao meu irmão, André, que me motiva através das suas conquistas alcançadas através de disciplina, estudo e perseverança.

Ao professor José Roberto Gomes ativo participante na elaboração deste trabalho, importante na minha vida não somente pelo conhecimento acadêmico transferido, mas acima de tudo pelo carinho, respeito e dedicação pelo trabalho e pelas pessoas que o cercavam.

À minha orientadora, Sandra Regina cuja disponibilidade, atenção e sugestões foram imprescindíveis para elaboração desta pesquisa.

Ao CNPq, a FAPERJ e a PUC-Rio, pelos auxílios concedidos, sem os quais este trabalho não poderia ter sido realizado.

Aos colegas do mestrado acadêmico, especialmente Juliana, Cristina, Monique, Isabel, Suzane, Lavilha, Danilo e Filipe pelo incentivo, ajuda, pelas conversas e momentos divertidos. 


\section{Resumo}

Lyra, Danielle Didier; Pinto, Sandra Regina da Rocha. Uma Análise Descritiva sobre o Processo de Transferência de Conhecimento dos Trabalhadores Maduros para os mais Jovens nas Organizações. Rio de Janeiro, 2010. 144p. Dissertação de Mestrado - Departamento de Administração, Pontifícia Universidade Católica do Rio de Janeiro.

O objetivo dessa pesquisa é investigar e descrever como a dinâmica de transmissão de conhecimento tem sido vivenciada especificamente pelos trabalhadores maduros, em duas empresas públicas. $\mathrm{O}$ trabalhador maduro costuma assumir um papel relevante dentro das organizações, já que seus anos de trabalho e experiência lhe renderam a aquisição de um tipo de conhecimento que envolve não somente aspectos técnicos e explícitos, mas também um componente tácito, que não é tão facilmente codificado e transmitido. Considerando ainda, que estes indivíduos se encontram perto da aposentadoria, sua capacidade de transmitir conhecimento acaba por refletir e determinar a capacidade da própria organização em reter e preservar sua memória organizacional. Para tanto, realizou-se dois estudos de caso com base em entrevistas semiestruturadas. Descobriu-se que ao longo da história das empresas em análise houve algumas iniciativas descontínuas orientadas para a estruturação do processo de transferência de conhecimento. Além disso, a análise do conteúdo das entrevistas realizadas revelou quatro categorias: a) conversão de conhecimento: processos, entraves e condições facilitadoras, b) relação entre a cultura e a aprendizagem organizacional, c) as percepções do olhar maduro sobre relações, papéis, postura do aprendiz e processo de aprendizado do aprendiz e d) oportunidades geradas pelo conhecimento adquirido. A principal dificuldade apontada pelos entrevistados diz respeito à falta de estruturação relacionada aos processos de gestão do conhecimento. Em consequência, predomina a passagem de conhecimento informal de modo que cada trabalhador maduro desenvolve sua própria metodologia de ensino.

\section{Palavras-chave}

Gestão do conhecimento; memória organizacional; trabalhador maduro. 


\section{Abstract}

Lyra, Danielle Didier; Pinto, Sandra Regina da Rocha (Advisor). A Descriptive Analisis of the Knowledge Transfering Process from Mature Workers to Younger ones Inside Organizations Rio de Janeiro, 2010. 144p. MSc. Dissertation - Departamento de Administração, Pontifícia Universidade Católica do Rio de Janeiro.

The aim of this research is to investigate and describe the knowledge transmission dynamic as experienced by senior workers in two public companies. Senior workers usually take up an important role in companies because the years of experience gained at work resulted in the acquisition of knowledge not only related to technical and explicit aspects, but also to a tacit dimension not easily codified and passed on. Taking also into consideration that these workers are close to retirement, their ability to pass on knowledge reflects and determines the capability of the company itself to keep and protect its organizational memory. In order to carry out this research, two case studies based on semi-structured interviews led to the conclusion that throughout the concerned companies' histories there were discontinued initiatives related to the knowledge transmission process. In addition, the analysis of the interviews' contents showed the existence of four categories: a) conversion of knowledge: processes, obstacles and enabling conditions, b) relationship between culture and organizational learning, c) perception of seniors towards relations, roles, apprentice's attitude and learning process, and d) opportunities created by the knowledge acquired. The major difficulty pointed out by the interviewees is connected to the lack of structuring as far as the knowledge management processes are concerned. Therefore, the informal knowledge transmission prevails and, as consequence, each senior worker develops his own teaching methodology.

\section{Keywords}

Knowledge management; organizational memory; senior worker. 


\section{Sumário}

1. Descrição do Problema 12

1.1. Introdução 12

$\begin{array}{lr}1.2 . \text { Objetivos } & 15\end{array}$

1.3. Relevância 16

1.4. Delimitação do estudo 16

1.5. Estrutura da Dissertação 18

2. Revisão da Literatura 19

2.1. Categorização dos trabalhadores maduros 19

2.1.1. Trabalhador maduro no mercado de trabalho 21

2.2. Gestão do conhecimento 25

2.2.1. Tipos de conhecimento 25

2.2.2. A criação contínua de conhecimento nas empresas 26

$\begin{array}{ll}2.2 .3 \text {. Captura de conhecimento } & 29\end{array}$

2.2.4. Transferência de conhecimento 31

2.2.5. Aprendizagem organizacional e individual 33

2.2.6. Criando uma cultura de aprendizagem 38

2.3. Memória Organizacional 43

2.4. Gestão do Conhecimento na Administração Pública 45

2.4.1. Pesquisa IPEA $2005 \quad 46$

2.4.2. Principais Facilitadores $\quad 48$

2.4.3. Principais Entraves 49

2.4.4. Práticas de Gestão do Conhecimento relacionados

2.5. Proposições da pesquisa $\quad 54$

3. Metodologia $\quad 56$

3.1. Tipo de Pesquisa 56

3.2. Coleta e Análise dos Dados 61

3.3. Limitações 66

4. Análise e Discussão dos Resultados 68

4.1. O BNDES 68

4.1.1. Conversão de conhecimento: iniciativas, processos, entraves e condições facilitadoras $\quad 70$

4.1.2. Relação entre a cultura e a aprendizagem organizacional $\quad 84$

4.1.3. As percepções do olhar maduro $\quad 87$

4.1.4. Oportunidades geradas pelo conhecimento adquirido 96

4.2. A Empresa $F \quad 98$

4.2.1. Conversão de conhecimento: iniciativas, processos, entraves e condições facilitadoras 99

4.2.2. Relação entre a cultura e a aprendizagem organizacional 111

4.2.3. As percepções do olhar maduro 113

4.2.4. Oportunidades geradas pelo conhecimento adquirido 121 
5. Conclusão 123

5.1. Considerações Finais 132

6. Referências Bibliográficas 134

$\begin{array}{ll}\text { 7. Anexos } & 141\end{array}$

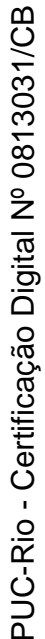




\section{Lista de tabelas}

Tabela 1 - Atritos da Transferência de Conhecimento

Tabela 2 - Estratégias de pesquisa de acordo

com as situações indicadas

Tabela 3 - Entrevistados BNDES

63

Tabela 4 - Entrevistados Empresa $\mathrm{F}$ 


\section{Lista de figuras}

Figura 1 - Modelo SECI da Criação do Conhecimento 28

Figura 2 - Processo de Aprendizagem Experimental 37

Figura 3 - Práticas de Gestão de Conhecimento 47

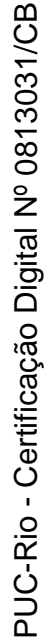




\section{Lista de gráfico}

Gráfico 1- Práticas na área de Recursos Humanos

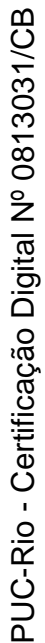

